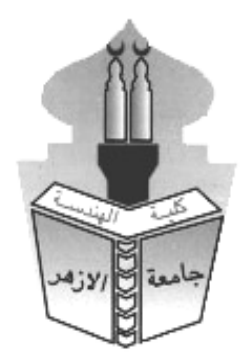

\title{
NOVEL SENSORS OF THE LINEAR DISPLACEMENT MEASUREMENT WITH HIGH-RESOLUTION FOR MECHATRONICS APPLICATIONS
}

\author{
Amir R. Ali ${ }^{* 1}$, and Momen Algouhary ${ }^{1}$ \\ ${ }^{1}$ Applied-Science \& Robotics Laboratory for Applied-Mechatronics (ARAtronics Lab.); \\ Mechatronics Engineering Department, Faculty of Engineering and Materials Science, German \\ University in Cairo (GUC), New Cairo, 11835, Egypt \\ *Corresponding Author E-mail: amir.ali@guc.edu.eg \\ Received :6 Oct. 2021 Accepted:4 Nov. 2021
}

\begin{abstract}
A displacement sensor with high resolution is presented in this paper using an elastic sphere that acts as the sensing element; which can be used in several applications of mechatronics. To have the elastic behavior for the sensing element, the sphere was made from polymeric material with $600 \mu \mathrm{m}$ in diameter. The sphere vibrates mechanically in response to a time varying displacement excited by piezo-stack actuator (PZT). The mechanical vibration transmitted to the elastic sphere was monitored optically based on tracking the optical resonance shifts on the transmission spectrum, in turn the displacement of the piezo-stack actuator can be determined. The sphere was lying between two micro-sticks made from silica with $\sim 80 \mu \mathrm{m}$ in diameter for each with $\sim 3 \mathrm{~cm}$ long; one of them is fixed while the other is mechanically connected to the PZT. By moving the PZT, the displacement was fully transmitted to the sensing element leading to a change in its morphology and therefore shifts of its optical resonances. By tracking these shifts and comparing them with typical electronic strain gauges used within piezo-nanopositioners, it is possible to determine the displacement of the PZT in high resolution. The resolution for the new design was recorded to be up to $\sim 3.16 \mathrm{~nm}$ with sensitivity up to $\sim 2 \mathrm{~nm} / \mu \mathrm{m}$. The novelty for the proposed design could be used for several mechatronics applications like the feedback on the linear actuators for the micro/nano robots, and also it can extend for structural health monitoring in nanoscale.
\end{abstract}

Keywords: Displacement measurement; high resolution sensor; mechanical vibration; piezo-stack actuator; force transmissibility. 


\title{
مستشعرات جليدة لقياس التحركات الخطية بدقة عالية لتطبيقات هندسة الميكاترونيات
}

\author{
أمير رشدى على ، مؤمن الجوهرى \\ الجامعة الالمانية_ القاهرة \\ *Corresponding Author E-mail: amir.ali@guc.edu.eg
}

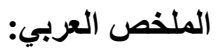

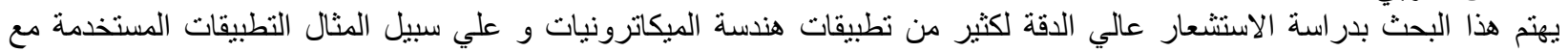

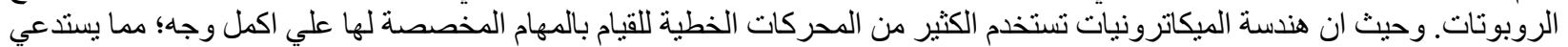

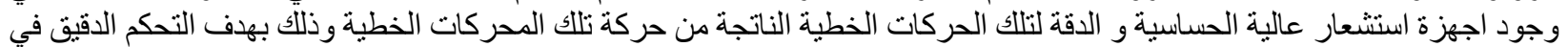

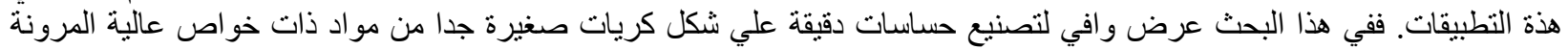

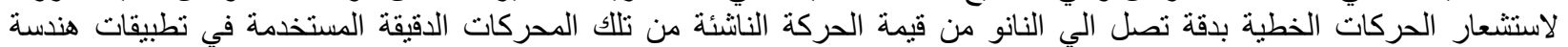

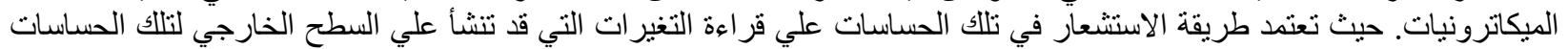

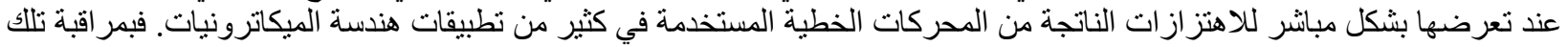

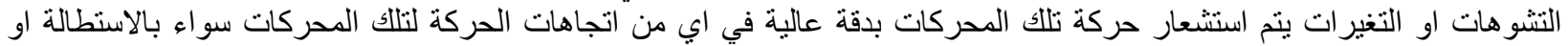

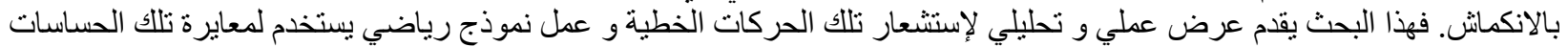

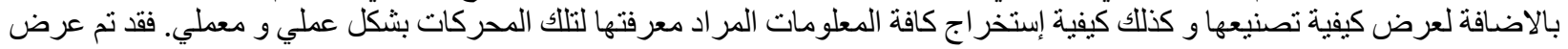

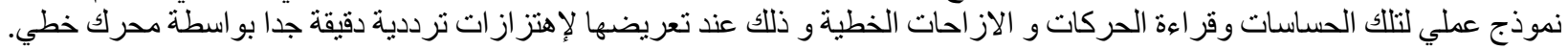

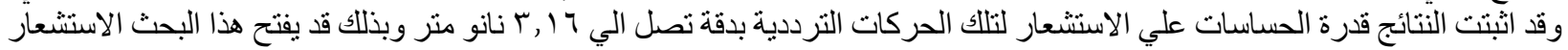

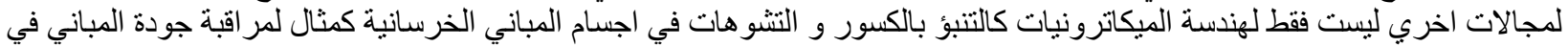
الاعمال الانشائية للهندسة المدنية.
\end{abstract}

\section{NOMENCLATURE}

$d \quad$ Distance between the partially reflected surfaces

$n \quad$ Index of refraction of the sphere

$\Delta n \quad$ Changes in the index of refraction

$p \quad$ The multiple integer

$Q \quad$ The quality factor

$r \quad$ Radius of the sphere

$\Delta r \quad$ Deformation in the radius of the elastic sphere

$x \quad$ Original value for the radius of the sphere

$\Delta x \quad$ Vibration input from the Piezo-stack

$\varepsilon_{r} \quad$ Strain along the polar direction of the sphere

$\varepsilon_{x} \quad$ Axial strain along the $x$-direction

$\lambda$ Wavelength of the light

$\Delta \lambda$ Resonance optical shifts of the wavelength

$\frac{d \lambda}{d x} \quad$ Sensitivity of the elastic sphere

$v \quad$ Poisson's ratio

$\sigma_{y} \quad$ Standard deviation 


\section{INTRODUCTION AND THEORY OF OPERATION}

Recently, the exact measurement of motions on the nanometer scale, either directly or indirectly, involves many applications in technology [1,2]. Nano-positioners, the microfabrication industry, vibration isolation devices, calibration equipment, surface topography, and measurements of thin film are examples of the importance of measuring motions and displacements on the nanoscale. Nowadays, in the mechanical nano-manufacturing facilities and laboratories, nanometer displacement sensors have become very essential. Much work has been done on the growth of nano-sensors in general and displacement sensors in particular [3]. Several techniques have therefore been developed to address this problem, including mechanical sensors [4-7], highperformance capacitive sensors [8-12], Eddy-current sensors [13-15], laser interferometers and optical encoders [16-21]. The high quality factor $(Q)$ for the techniques that are depending on any of the optical metrology methods made it a powerful tool for detection that can be used in many different fields [22]. In this paper, an elastic micro-sphere was basically used as the sensing element to detect the displacement of piezo-stack on the nanoscale. Mechanical vibrations generated by the piezo-stack were fully transmitted to the elastic sphere through the micro silicastick. By monitoring these vibrations on the elastic sphere optically through tracking its optical resonance shifts on the transmission spectrum, in turn the displacement of the piezo-stack actuator can be determined.

To start tracking the optical shifts due to the mechanical vibration, the optical resonance must be occur first and resonate inside the optical resonator [23]. Figure 1 shows the optical resonance condition occurs in a planar resonator in figure 1a versus the spherical resonator in figure $1 \mathrm{~b}$. In general; the optical resonance condition occurs in any waveguide once the travel distance of the light through the waveguide becomes equal to the multiple integer $p$, of its wavelength $\lambda$, the optical resonance condition will be satisfied and will be seen as a sharp dips on the transmission spectrum [24-29] as clear in figure 1c. In this paper the waveguide that used, was the microspherical elastic resonator similar to the schematic shown in figure $1 \mathrm{~b}$, so the travel distance of the light through it was equal to $2 \pi r$, where the $r$, is the radius of the sphere. Equation 1 shows that the optical resonance that used in this paper will be satisfy when the following condition occurs

$2 \pi r n \cong p \lambda$

where is $n$, is the index of refraction. From equation 1 it is clear that any minute change caused by the mechanical vibration coming from the piezo-stack and perturbs the radius of the elastic sphere by, $\Delta r$ will lead to perturb the optical shifts by, $\Delta \lambda$ on the transmission spectrum and this relation could be described in equation 2 .

$\frac{\Delta \lambda}{\lambda}=\frac{\Delta r}{r}+\frac{\Delta n}{n}$

where $\Delta n$ is the changes of the index of refraction and it has almost no effect because the dominant effect will be due to the mechanical deformation on the radius on the elastic sphere, $\Delta r$.

Figure 1d shows a block diagram for the feedback loop of the experimental setup that was used in the paper. The piezo-stack provides the vibration to drive a mechanical displacement, $\Delta x$ in the $x$ direction as an input for the proposed sensor. Due to the opto-mechanical coupling, mechanical vibration perturbs the radius of the elastic sphere [30,31]. In turn, tunes the optical modes of the optical resonance for the sphere on the transmission spectrum with $\Delta \lambda$, wherein $\Delta \lambda$ contains 
information on the mechanical displacement, $\Delta x$. Finally, the piezo driver controller was using the electronic strain gauges within piezo-nanopositioners to close the feedback loop for the mechanical input.

\section{(a)}

(b)

Optical fiber

From the

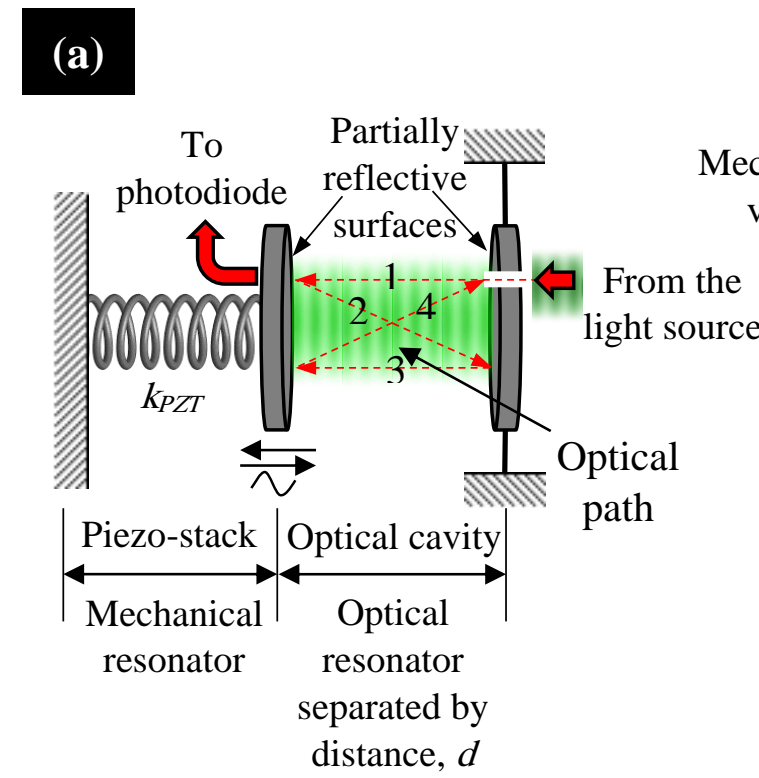

Travel distance of the light $\sim 4 d$

$\triangleright$ Optical resonance condition occurs when

Techanical resonator $(\mathrm{PZT})$

vibrates in $x$-direction

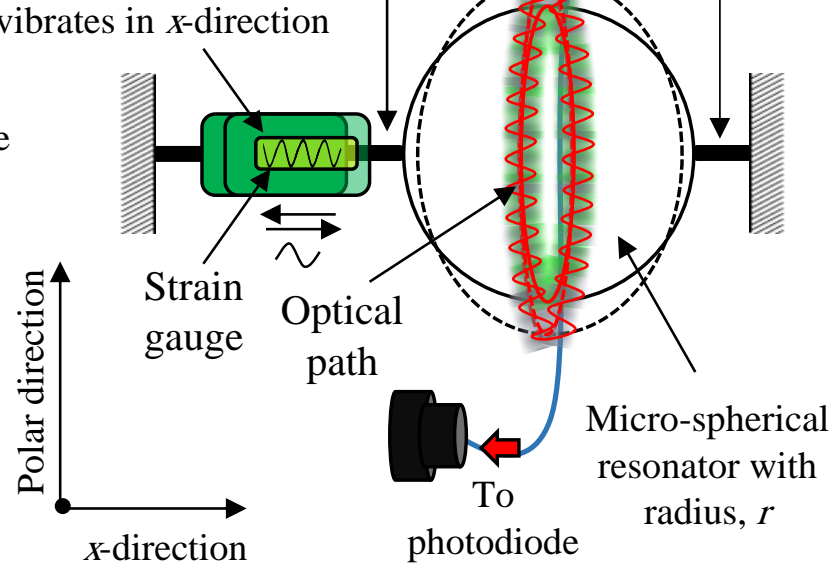

Travel distance of the light $\sim 2 \pi r$

$\triangleright$ Optical resonance condition occurs when

$$
\begin{gathered}
4 d n \cong p \lambda \\
\frac{\Delta \lambda}{\lambda}=\frac{\Delta d}{d}+\frac{\Delta n^{\prime} \text { Negligible }}{\underbrace{\prime}_{i} n}
\end{gathered}
$$

(c)

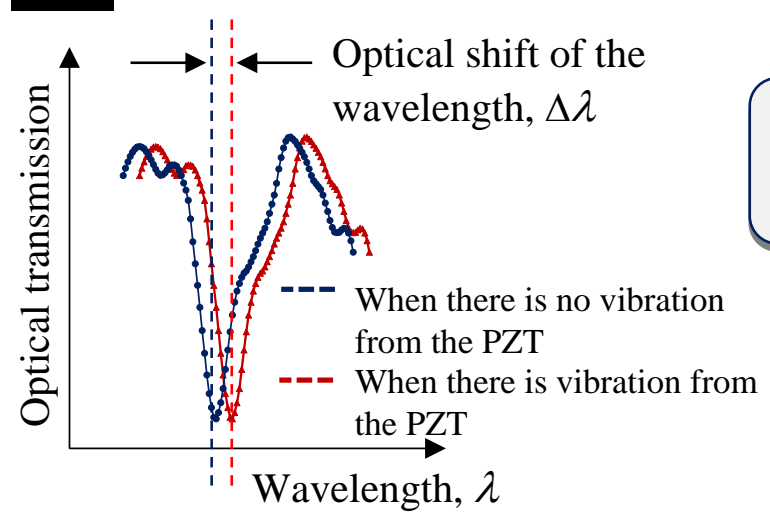

(d)

$$
\begin{gathered}
2 \pi r n \cong p \lambda \\
\frac{\Delta \lambda}{\lambda}=\frac{\Delta r}{r}+\frac{\Delta x^{\prime}}{i}{ }^{\prime} \text { Negligible }
\end{gathered}
$$

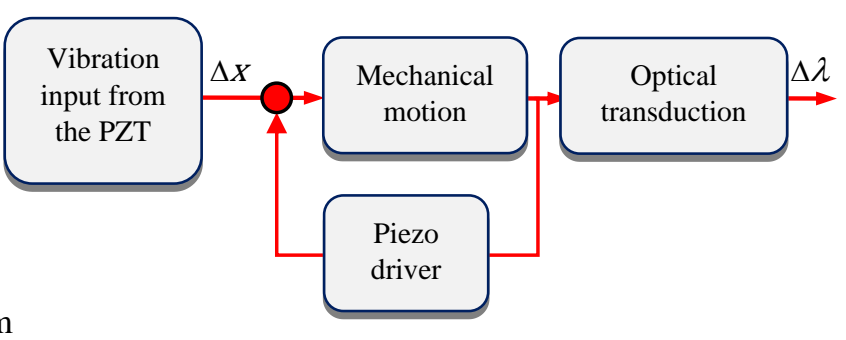

$\Delta \lambda \alpha \Delta x$

Fig. (1): Schematic for, (a) the planar resonator that acts a linear waveguide which contains from pair of partially reflected surfaces, (b) the micro elastic spherical resonator that used as a sensor in this paper as a 


\section{NOVEL SENSORS OF THE LINEAR DISPLACEMENT MEASUREMENT WITH HIGH- RESOLUTION FOR MECHATRONICS APPLICATIONS}

spherical waveguide with radius $r$, (c) the transmission spectrum that will be detected using the photodiode, (d) the block diagram for the feedback loop of the experimental setup that was used to measure the displacement.

So figure 1 shows as schematic for the optical resonance conditions when the light with wavelength, $\lambda$ was circumnavigates inside a high quality factor optical waveguides (planar and spherical). Meanwhile, the concept of the spherical optical waveguide shown in figure $1 \mathrm{~b}$ can be understood as part of the sphere can be represented as a pair of partially reflective glass optical flats spaced with distance $d$, part, with the reflective surfaces facing each other as you can see in figure 1a. The light emitted from a point on the source and only one ray is traced. As the ray passes through the paired flats, it is repeatedly reflected to produce multiple transmitted rays which are collected by the focusing lens or photodiode and brought to the transmission spectrum on the computer as clear in figure 1c. On the other side the piezo-stack that acts as a mechanical oscillator can be represented with a spring constant KPZT. As seen in figure $1 \mathrm{a}$ and $1 \mathrm{~b}$ both of the mechanical and optical cavities are mechanically connected together. The motion of the piezo-stack in the $x$ direction brings a vibration in the optical path length to alter the cavity resonance, $\Delta \lambda$ which in turn causes a shift on the optical resonance on the transmission spectrum. Now, any minute change in the morphology of the elastic-sphere caused by the displacement generated from the piezo-stack will cause a change in the wavelength of the light. Due to the high quality factor that optical techniques can reach, we will exploit that factor in this paper to detect and measure the mechanical displacement of the piezo-stack with high resolution. Based on that, the proposed sensor design can enhance the overall control process for the mechatronics applications that require a very highresolution for the linear motion feedback. The applications that can use the proposed sensing technique will be; the Stewart platform, delta robots, gantry robots and the linear prismatic joint at the end-effector mechanism in the SCARA robots. Controlling all these types of robots using high-resolution sensing elements for the feedback will definitely enhance the closed loop feedback on the systems.

\section{ANALYSIS OF THE PROPOSED SENSOR}

In this paper the sensing element was an elastic sphere subjected to mechanical strain using piezostack actuator (PZT). The sphere is connected between two micro silica sticks, one of them is fixed in the base while the other is connected to the PZT. When moving the PZT; the vibration of the mechanical forces will be transmitted to the elastic sphere. The response of the elastic sphere can be detected optically, by monitoring the shifts on the optical resonances (modes) through the transmission spectrum. The change of the optical modes will be depending on the elastic deformation in the polar direction of the sphere, so the changes of the index of refraction in equation 2 will be negligible $[32,33]$ as also clear in figure $1 b$. Tracking the shifts of the optical modes in the polar direction of the sphere can lead to determining the displacement of the PZT. The strain along the $x$-direction that is generated using the PZT is given by $\varepsilon_{x}=\Delta x / x$, where $\Delta x$ is the mechanical displacement of the PZT in $x$-direction and, $x$ will have the same value for the radius of the sphere, $r$. The strain of the elastic sphere in the polar direction that is induced by the changes in displacement of the PZT is proportional to the application of a force from the PZT on the $x$-direction and it is given by $\varepsilon_{r}=\Delta r / r$, where $\Delta r$ is the change of the sphere's radius with respect to the original radius, $r$. The ratio between the polar stain, $\varepsilon_{r}$ and the axial stain, $\varepsilon_{x}$ is governed by Poisson's ratio, $v=-\varepsilon_{r} / \varepsilon_{x}$ [34]. Therefore, the optical shift, $\Delta \lambda$ from equation 2 can be written as a function of the displacement of the PZT for small displacement as 
$\Delta \lambda / \lambda=-v \varepsilon_{x}$ and then $\Delta \lambda=-\lambda v \varepsilon_{x}$. From this relation, the change in the mechanical displacement act upon the PZT can be written in equation 3 as follows:

$$
\Delta x=-\frac{r}{\lambda v} \Delta \lambda
$$

So based on equation 3 the proposed design for the displacement measurements can be used not only for mechanical sensing applications but also can be extended to the biosensing applications [35].

\section{MATERIAL AND EXPERIMENTAL METHODS}

\subsection{Fabrication of The Sensing Element}

A mixture of silicon base and crosslinker of the polydimethylsiloxane (PDMS)-base was used to fabricate the elastic micro-sphere that acts as the sensing element in this paper. The ratio between the silicon polymeric base to the crosslinker was 60:1, 60 parts of the polymeric base and one part of the crosslinker, by volume with Poisson's ratio $(v=0.49)$ [17]. In figure 2 , the elastic-sphere was fabricated with $600 \pm 20 \mu \mathrm{m}$ in diameter using this mixture right after the end of mixing process when the mixture takes the consistency of a fluid with high viscosity. The mixture was injected between two micro silica sticks with $\sim 80 \mu \mathrm{m}$ in diameter with $\sim 3 \mathrm{~cm}$ long optical fibers. The injection process was depending on using typical commercially available syringes. Due to the surface tension force and gravity the micro-sphere was formed between the tips of the optical fibers. After heating the mixture of the PDMS 60:1 on the top of the optical fibers in the oven at $100^{\circ} \mathrm{C}$ for 4 hours, it becomes elastic with a spherical shape and ready to use in the experiment. One of the optical fibers was fixed on the translation stage while the other fiber is mechanically connected to the piezo-stack (PZT) that acts as an input source of displacement. In turn, this displacement was transmitted to the elastic micro-sphere and changed its morphology. Optical tracking for these morphologically changed on the sphere leads to be used as a displacement sensor with high-resolution. 


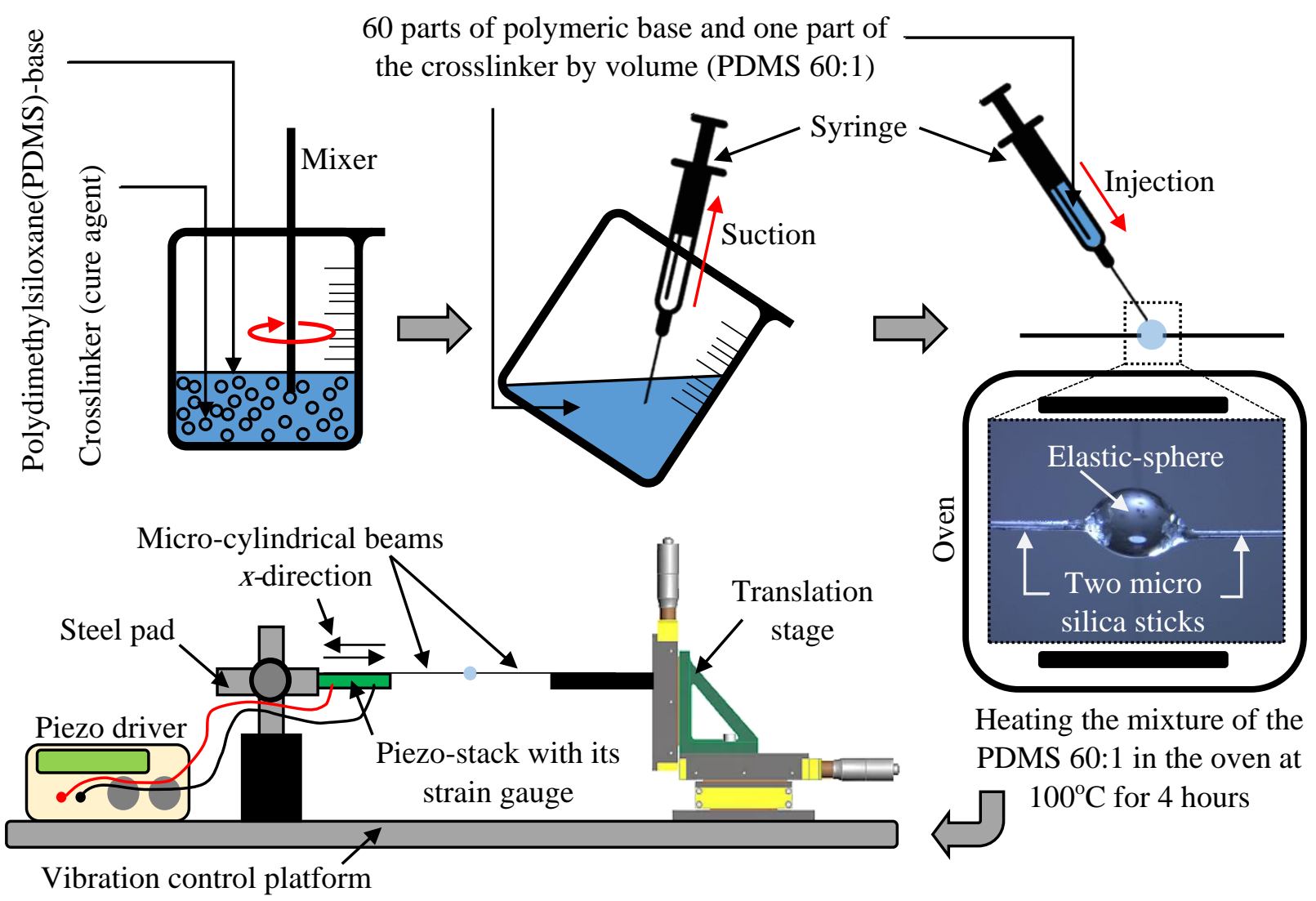

Fig. (2): Schematic and photograph for the fabrication process of the elastic micro-sphere with $600 \pm 20 \mu \mathrm{m}$ in diameter using 60 parts of polymeric base and one part of the crosslinker by volume (PDMS 60:1).

\subsection{Experimental Setup}

A schematic for the displacement measurement experiment using the micro-elastic sphere is shown in figure 3 . The nominal wavelength, $\lambda$ of the used laser was $\sim 1314.6 \mathrm{~nm}$. The output of the laser diode is connected to a single-mode optical fiber. In order to monitor the morphology change on the micro-sphere efficiently, optical coupling between the sphere and the light should be occur. This may be achieved by melting the protective buffer layer using a micro-torch to create a tapered section to let the light propagate from the optical fiber to the elastic sphere by mechanically coupling (based on Van Der Waals force) [30]. Once the round trip of the light circumnavigates through the sphere it returns back to the optical fiber and the intensity of the light will be seen using the photodiode. The piezo-stack (Thorlabs PZS001) with the typical electronic strain gauges connected on it was used to measure the displacement that was generated from the piezo driver and then compared to the shifts of the transmission spectrum for the micro-sphere. The host personal computer (PC) performs the scanning, data acquisition and analysis. A software module, developed in-house, identifies the optical resonance of the sphere in the transmission spectrum and monitors their shifts. It provides a reliable and fast fit to an experimental spectrum and enables a continuous tracking and recording of resonance shifts in real time. 


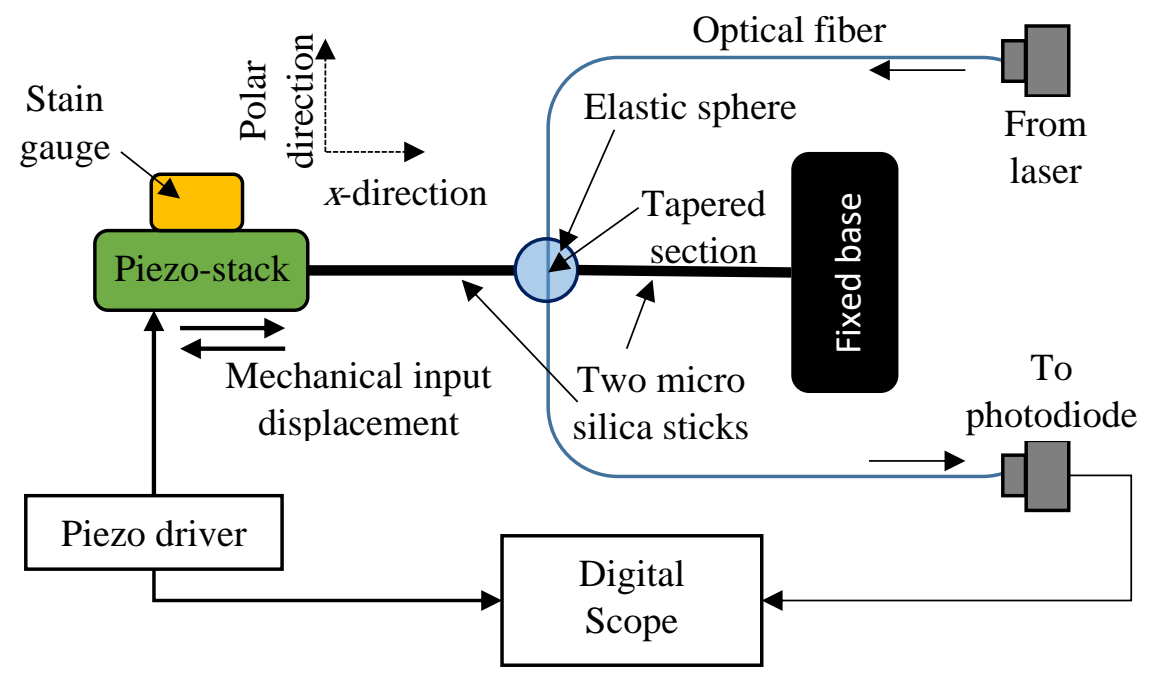

Fig. (3): Schematic of the displacement measurement experiment using the micro-elastic sphere.

A photograph for the elastic microsphere and its setup is shown in figure 4. The elastic sphere is fabricated between two micro-sticks made from silica. One of them is connected to the fixed base while the other is connected to the source of the displacement which is the piezo stack. The displacement is mechanically transmitted to the sphere by using the PZT that vibrates the sphere in the direction perpendicular to the tapered section of the optical fiber. Then the circumferential optical mode shifts on the equatorial plane are tracked. The piezo driver is used to control the piezo stack and provide a harmonic vibration on the elastic sphere with a fixed amplitude.

Figure 5 shows the transmission spectrum of the elastic micro-sphere when the optical resonance contention satisfies and when there is no displacement coming from the piezo stack. The observed quality factor for this experiment was $\sim 1.3 \times 10^{6}$. When the displacement of the piezo-stack was changed in turn, the transmission spectrum will change as seen in figure 6a. Significance of evolution shifts of the dip wavelength is observed when the piezo-stack increases the amplitude of the displacement on the elastic sphere. Figure $6 \mathrm{~b}$ shows the direct relation between the changes in the transmission spectrum of the elastic sphere and the displacement varying from $0.00412 \mu \mathrm{m}$ to $0.02064 \mu \mathrm{m}$. The slope of the best linear fitting gives the displacement sensitivity of $\sim 2 \mathrm{~nm} / \mu \mathrm{m}$ as seen in figure $6 b$. 


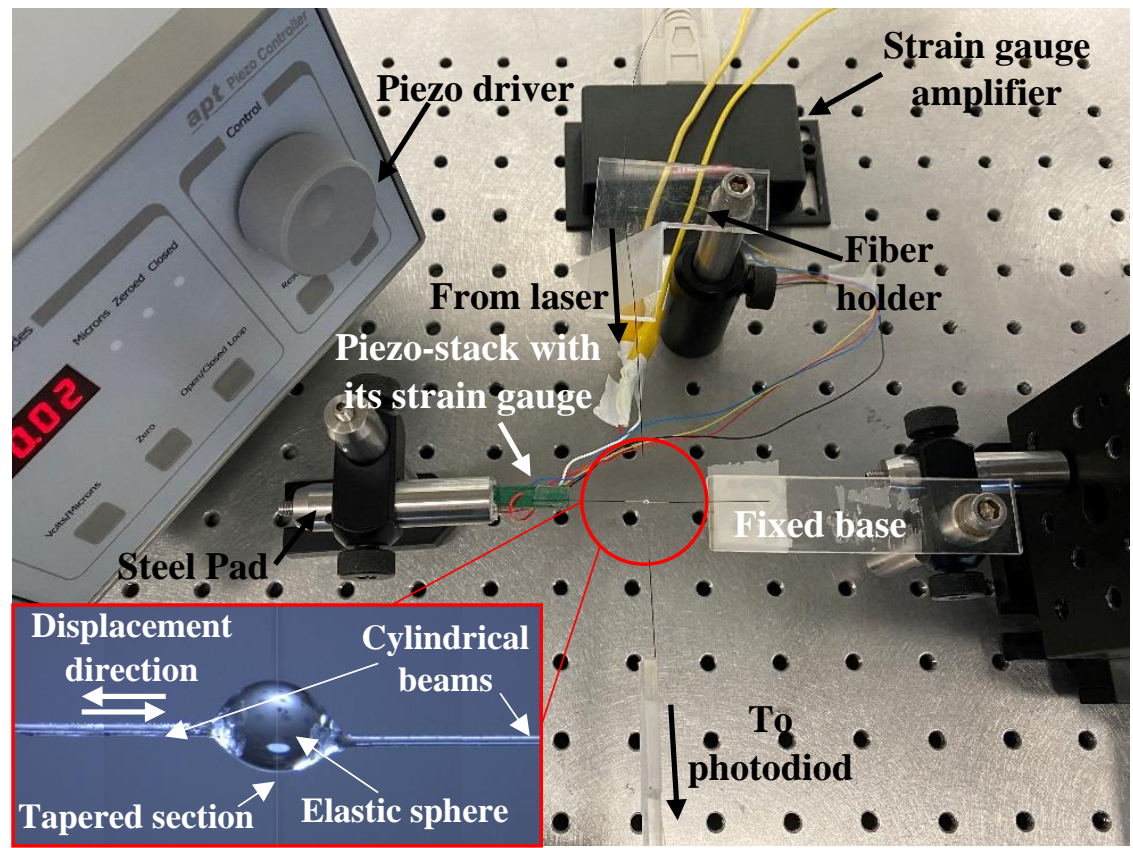

Fig. (4): Photograph and close-up of the elastic micro-sphere that was used in the displacement measurement experiment.

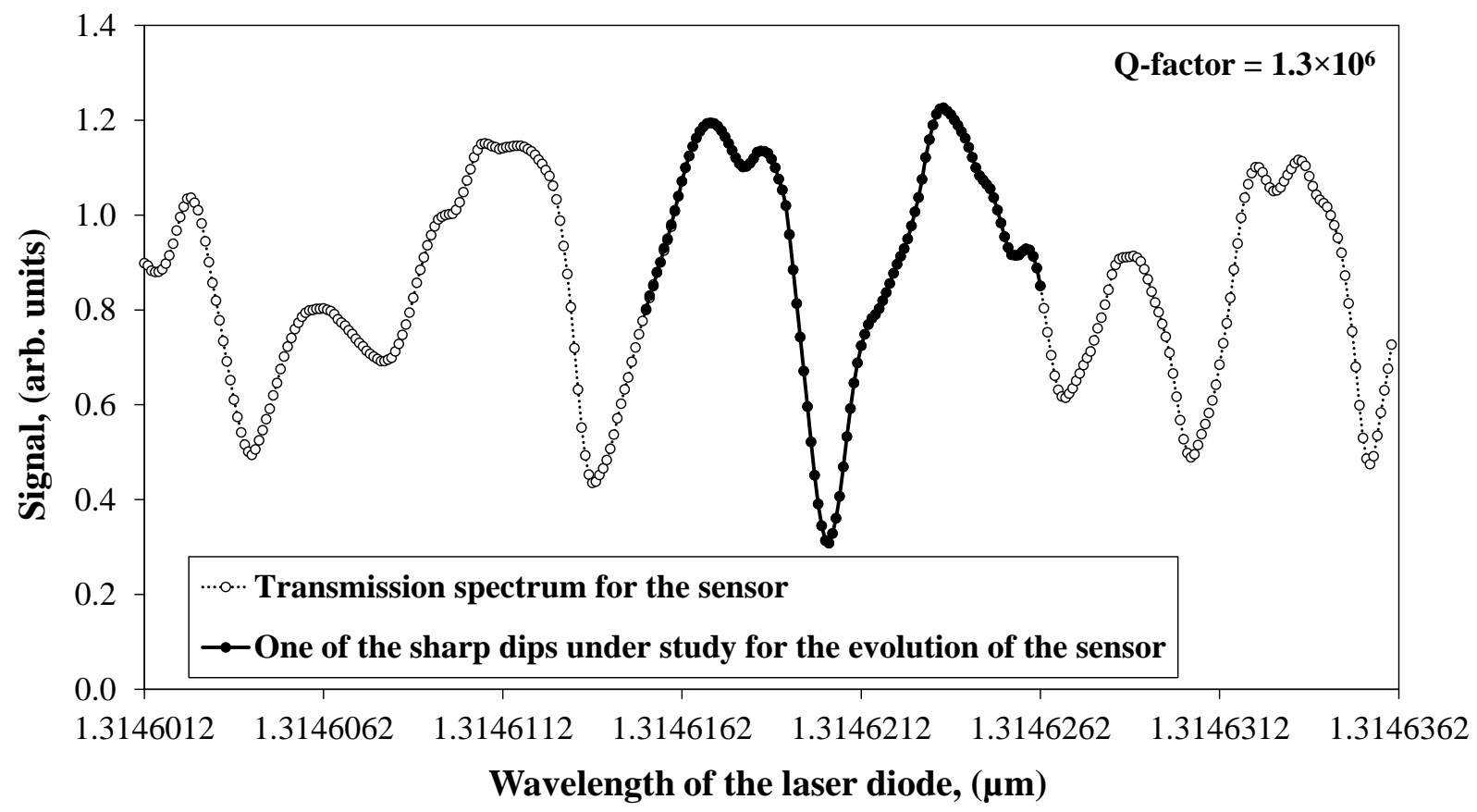

Fig. (5): Transmission spectrum of the proposed sensor with quality factor $1.3 \times 10^{6}$. 


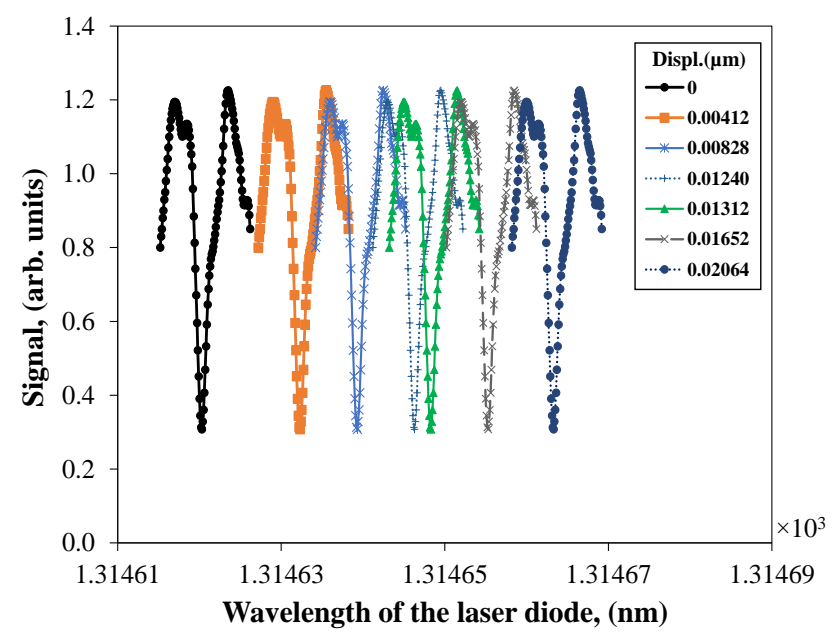

(a)

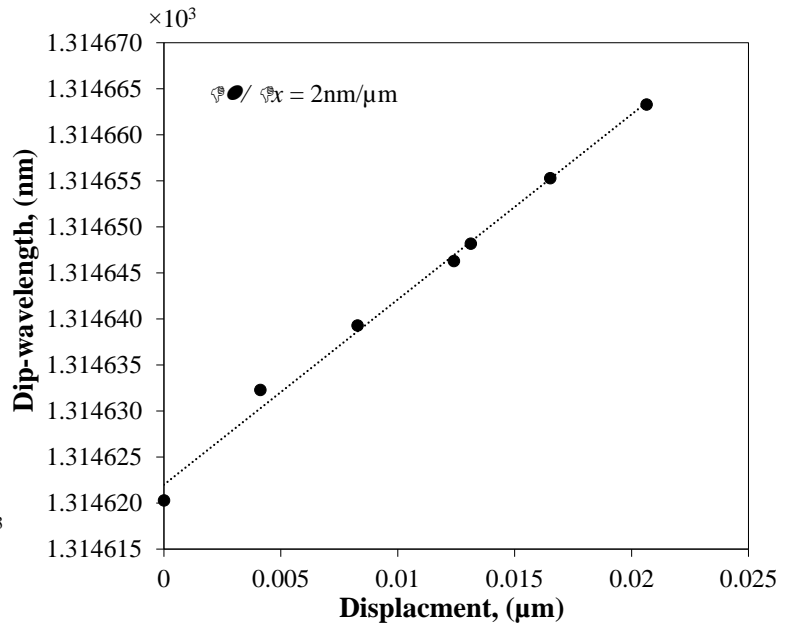

(b)

Fig. (6): (a) Transmission spectrum evolution of the sensor when the displacement changes and (b) Wavelength shifts of the elastic sphere as a function of displacement.

\section{RESULTS AND DISCUSSION}

The measurement in figure 7 shows the step response of the elastic sphere (sensing element) with $\sim 600 \mu \mathrm{m}$ in diameter via an optical technique and via the typical electronic strain gauges used within the piezo-stack. The direct relation between the optical shifts on the transmission spectrum and the mechanical displacement caused by the piezo-stack can be easily seen in figure 7 . The displacement was controlled to increase gradually with $0.02 \mu \mathrm{m}$ every 10 seconds using a closed loop algorithm from the piezo driver. It was clear that the optical shifts are following the mechanical displacement in the $x$-direction in a perfect manner and that proves the sensor's reliability and stability. As clear in figure 7, a very stable response from the sensor was observed under different displacement conditions and compared with typical electronic strain gauges used

within piezo-nanopositioners. The elastic sphere showed maximum wavelength deviations during the complete measurement cycle of $\sim 40 \mathrm{pm}, \sim 80 \mathrm{pm}, \sim 120 \mathrm{pm}, \sim 160 \mathrm{pm}$, and $\sim 200 \mathrm{pm}$ corresponding to the different displacement of $0.02 \mu \mathrm{m}, 0.04 \mu \mathrm{m}, 0.06 \mu \mathrm{m}, 0.08 \mu \mathrm{m}$, and $0.1 \mu \mathrm{m}$, respectively. 


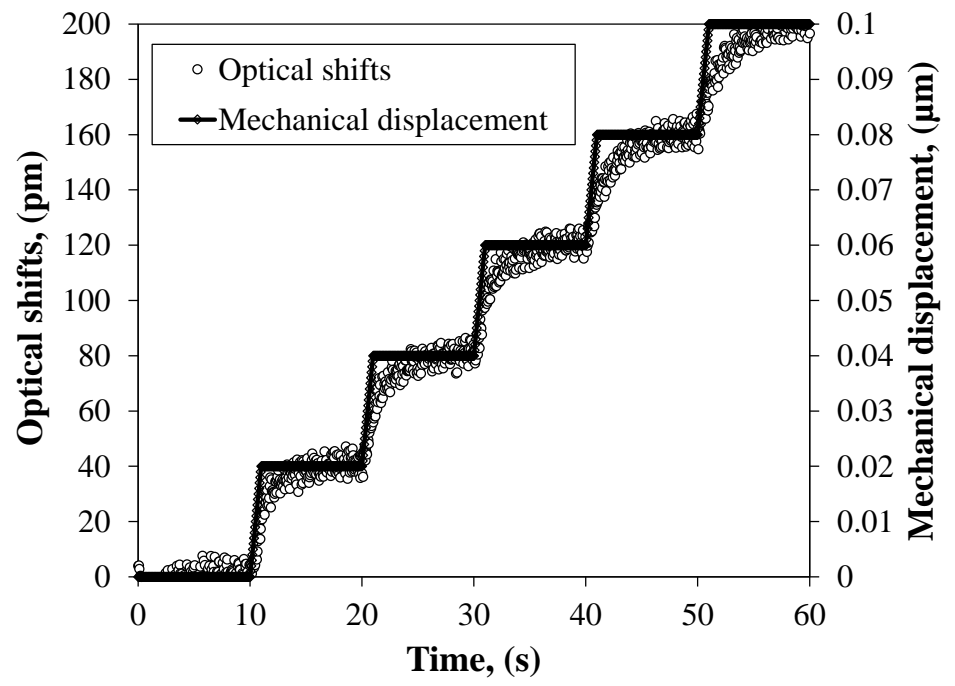

Fig. (7): Optical shift of the elastic sphere stepwise at given displacement.

Figure 8a shows the response for the elastic sphere under harmonic excitation at a frequency of $1 \mathrm{~Hz}$. The piezo-stack was harmonically driven with an amplitude of $0.02 \mu \mathrm{m}$ at law frequency $1 \mathrm{~Hz}$. Due to that, the elastic sphere was subjected to a harmonic loading and unloading at a frequency of $1 \mathrm{~Hz}$ and experiences a change on the optical shifts on the transmission spectrum harmonically with the same frequency and an amplitude of 40pm wavelength shifts. During this experiment, the mechanical displacement was also measured using the same typical electronic strain gauges used within piezo-stack. The optical shifts and the mechanical displacement are following each other very well. Calibration curve in figure $8 \mathrm{~b}$ shows the linear relation between the optical shifts of the elastic sphere and the mechanical displacement induced by the piezo-stack. The solid line is the best fit to the data and its slope represents the sensitivity of the proposed sensor. The sensitivity for the elastic sphere with $600 \mu \mathrm{m}$ in diameter was $d \lambda / d x \approx 2.0747 \mathrm{~nm} / \mu \mathrm{m}$ and the standard deviation was $\sigma_{y} \approx 6.563 \mathrm{pm}$. So, for this configuration using the optical shifts of the elastic sphere it could be measured as a mechanical displacement with a high resolution of $(d x / d \lambda) \sigma_{y}$ up to $3.16 \mathrm{~nm}$.

The displacement transmissibility from the linear actuator to the elastic micro-sphere through the micro-silica stick is addressing the novelty for the proposed sensor design. Because, it is the first time that the elastic micro-sphere which acts as the sensing element will not be in contact directly with the input. However, exploiting the use of the micro-silica stick for transmitting that displacement will help in sensing that linear motion in very high-resolution on the order of few nanometers as seen in the results section. The novelty also becomes clear from the results while using the proposed design to have better displacement sensitivity compared to those techniques that had been developed to address this problem using other types of resonators similar to the mechanical sensors [6,7], electrical sensors based on the capacitive transducers [9, 11, 12], Eddycurrent resonators [13-15], and laser interferometers [16-21]. 


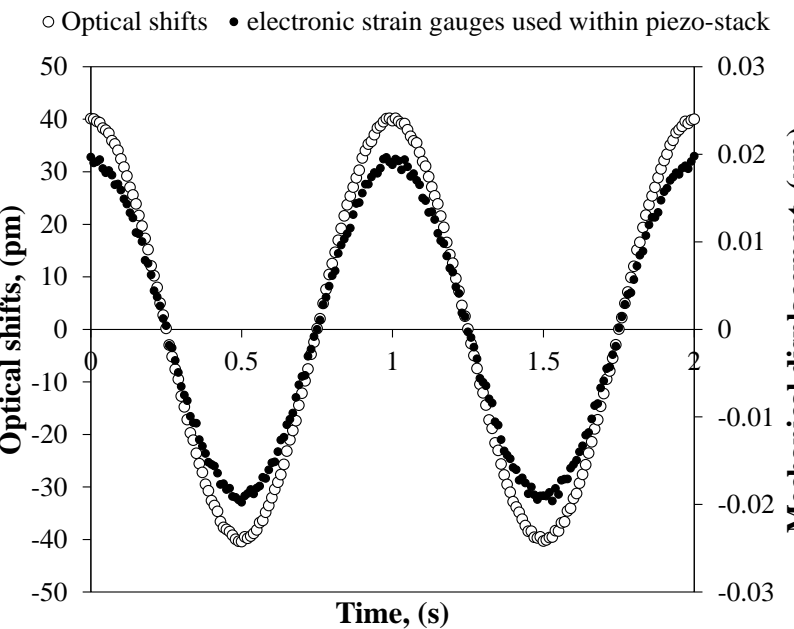

(a)

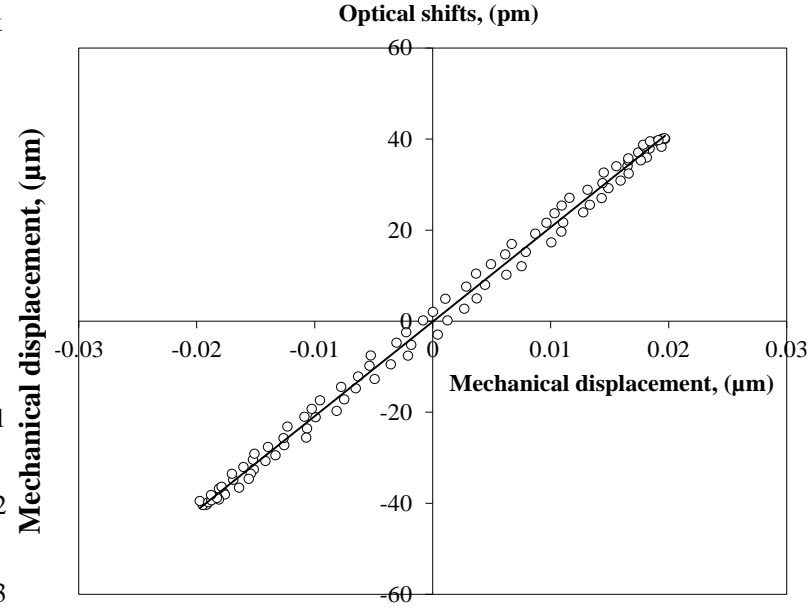

(b)

Fig. (8): (a) Optical shift of the elastic sphere versus the mechanical displacement recorded using the electronic strain gauge within the piezo-stack as a function of time at $1 \mathrm{~Hz}$ and (b) Displacement sensitivity of the elastic sphere.

In the above experiments the measurements were made at low frequency $(1 \mathrm{~Hz})$ of the displacement modulations and it shows a promising displacement sensitivity. So the next experiment shows the effect of the sensitivity on displacement modulation frequency. Figure 9 shows the frequency dependence of the proposed displacement sensor when a harmonic frequency modulated up to $1.4 \mathrm{kHz}$ is applied. In these experiments, the displacement amplitude was kept constant as the frequency is varied. The figure shows that the proposed sensor starts losing its sensitivity after $200 \mathrm{~Hz}$ for the range of investigated frequencies. This frequency study proves that the elastic sphere is mechanically over-damped and its sensitivity rapidly drops with increasing frequency [18]. So based on that the sensing element which is the elastic sphere can be classified as a mechanical over-damped second order system subjected to harmonic excitation. By using harder elastomers (like, lower base-to-crosslinker ratio mixtures), the mechanical overdamping may be avoided. However, this will cause a reduction in the displacement sensitivity.

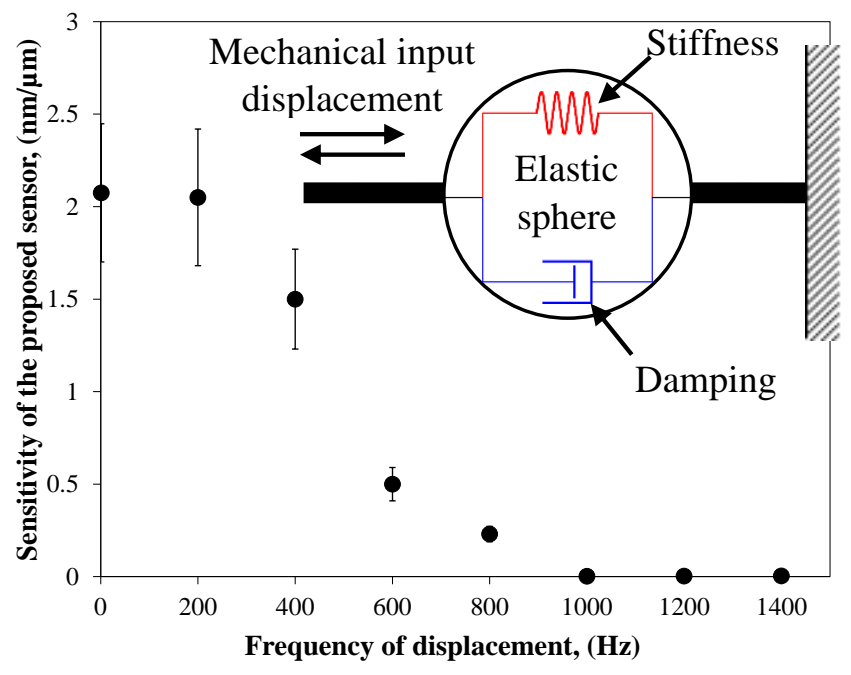




\section{NOVEL SENSORS OF THE LINEAR DISPLACEMENT MEASUREMENT WITH HIGH- RESOLUTION FOR MECHATRONICS APPLICATIONS}

Fig. (9): Sensitivity of the elastic sphere as function of harmonic displacement.

Figure 10 shows the effect of the size of the elastic sphere on the sensitivity for two different materials PDMS 60:1 and PDMS 10:1. As the results show in this figure, there is a dependency of the displacement sensitivity on the diameter of the elastic sphere for both materials. In this study, the displacement amplitude is kept constant for both materials (PDMS 60:1 and PDMS 10:1) as the diameter of the sphere is changed starting from $600 \mu \mathrm{m}$ to $1500 \mu \mathrm{m}$ in diameter. Results show that the sensitivity is affected by the sphere size and that is because the light starts to lose its intensity when the sphere becomes bigger and in turn losing its quality factor. So, based on that the smaller sphere will increase the displacement sensitivity.

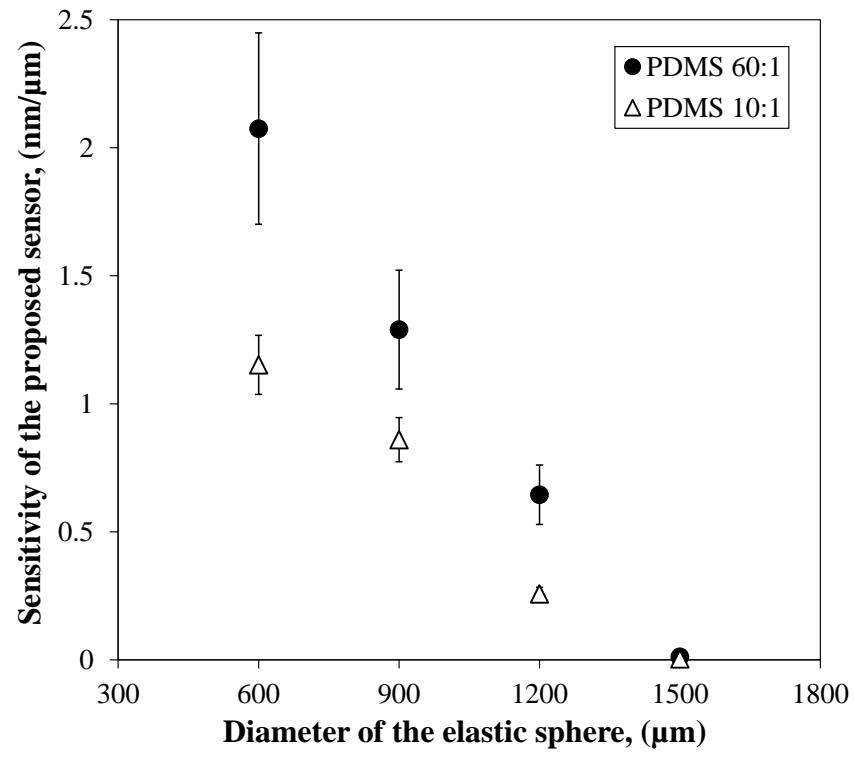

Fig. (10): Sensitivity of the displacement measurements as function of the size of the elastic sphere.

\section{CONCLUSIONS}

In summary, results show that the displacement can be measured with high resolution using a micro-elastic sphere. A mathematical model was developed throughout the experiments in order to characterize the new sensing design. A piezo-actuator was used in several experiments to induce displacement measurement. The measured displacement was done by tracking the optical resonance shifts of the elastic sphere through its transmission spectrum with high resolution due to the high quality factor that this proposed design can exhibit. The elastic sphere made from PDMS 60:1 provides high resolution for the displacement $(\sim 3.16 \mathrm{~nm})$ with sensitivity up to $(\sim 2 \mathrm{~nm} / \mu \mathrm{m})$. However, the sensor is mechanically over-damped and when increasing the displacement frequency the sensitivity drops after $200 \mathrm{~Hz}$ dramatically. This limits the use of the proposed sensor to the transient displacement detection at high frequencies. This problem can be avoided when using harder elastomers like the PDMS 10:1 (lower base-to-cure agent ratio mixtures) however it has a reduction in the displacement sensitivity as seen in the results above. On the other hand, using a softer material to fabricate the sensor leads to lower bandwidth and that is because the mechanical resonance of the sensor will decrease as well. However, softer material 


\section{NOVEL SENSORS OF THE LINEAR DISPLACEMENT MEASUREMENT WITH HIGH- RESOLUTION FOR MECHATRONICS APPLICATIONS}

will enhance the sensor sensitivity. Future work will focus on numerous new designs for the elastic sphere geometries, fabrication methods and materials will be investigated to improve mechanical bandwidth while maintaining sensitivity of the sensor. The proposed design for the displacement measurements can be used for many other mechanical sensing applications because of its simplicity of fabrication, reliability, compactness, its low cost and finally its transmissibility from the measuring phenomena to the elastic sphere.

\section{REFERENCES:}

[1] Zheng, H., Qiu,W., Gu, X., Zhang, Y., Zhu, W., Huang, B., Lu, H., Guan, H., Xiao, Y., Zhong, Y., Fang, J., Luo, Y., Zhang, J., Yu, J., Tittel, F., and Chen, Z.; "Accurate measurement of nanomechanical motion in a fiber-taper nano-optomechanical system”, Appl. Phys. Lett. 115, 013104, 2019.

[2] Lee, J., Wang, Y., Lin, Z., Lin, C., and Chan, C.; "Standing-wave interferometer based on single-layer $\mathrm{SiO}_{2}$ nano-sphere scattering" Opt. Express 25, 26628-26637, 2017.

[3] Liu, Y. and Higuchi, T., "Precision Positioning Device Utilizing Impact Force of Combined Piezo-Pneumatic Actuator," IEEE/ASME Transactions on Mechatronics, vol. 6, pp. 467-473, 2001.

[4] Fraden, J., Handbook of Modern Sensors: Physics, Designs and Applications Springer, 2010.

[5] Soloman, S., Sensors Handbook $2^{\text {nd }}$ ed. McGraw-Hill, 2009.

[6] Nyce, D. S., Linear Position Sensors: Theory and Application Wiley-IEEE, 2004.

[7] Gao, W., Precision Nanometrology: Sensors and Measuring Systems for Nanomanufacturing Springer, 2010.

[8] Gong, C., Chiu, H., Huang, L., Lin, C., Hsu, Z., and Tu, P., "Low-Cost Comb-Electrode Capacitive Sensing Device for Liquid-Level Measurement", Sensors Journal IEEE, vol. 16, no. 9, pp. 2896-2897, 2016.

[9] Khan, A., Islam, T., and Akhtar, J., "An Oscillator-Based Active Bridge Circuit for Interfacing Capacitive Sensors With Microcontroller Compatibility", Instrumentation and Measurement IEEE Transactions on, vol. 65, no. 11, pp. 2560-2568, 2016.

[10] Chetpattananondh, K., Tapoanoi, T., Phukpattaranont, P., and Jindapetch, N., "A self-calibration water level measurement using an interdigital capacitive sensor", Sensors and Actuators A: Physical, vol. 209, pp. 175, 2014.

[11] Jin, B., Zhang, Z., and Zhang, H., "Structure design and performance analysis of a coaxial cylindrical capacitive sensor for liquid-level measurement", Sensors and Actuators A: Physical, vol. 223, pp. 84, 2015.

[12] Baxter, L. K., Capacitive Sensors: Design and Applications IEEE, 1997.

[13] Yin, W., Binns, R., Dickinson, S. J., Davis C., and Peyton, A. J., "Analysis of the Liftoff Effect of Phase Spectra for Eddy Current Sensors" in IEEE Transactions on Instrumentation and Measurement, vol. 56, no. 6, pp. 2775-2781, Dec. 2007.

[14] Yang, H. C., and Tai, C. C., "Pulsed eddy-current measurement of a conducting coating on a magnetic metal plate", Meas. Sci. Technol., vol. 13, no. 8, pp. 1259-1265, Aug. 2002.

[15] Sophian, A., Tian, G. Y., Talyor D., and Rudlin, J., "Design of a pulsed eddy current sensor for detection of defects in aircraft lap-joints", Sens. Actuators A Phys., vol. 101, no. 1/2, pp. 92-98, Sep. 2002.

[16] Ioppolo, T., Kozhevnikov, M., Stepaniuk, V., Ötügen, V., and Sheverev, V., "A micro-optical force sensor concept based on whispering gallery mode resonators," Appl. Opt. 47 3009, 2008.

[17] Ali A.R., and Ioppolo, T., "Effect of Angular Velocity on Sensors Based on Morphology Dependent Resonances," Sensors. 14 7041, 2014.

[18] Ali, A.R., Ioppolo, T., Ötügen, V., Christensen, M., and Macfarlane, D., "Photonic electric field sensor based on polymeric microspheres," Journal of Polymer Science Part B: Polymer Physics 52, 276-279, 2013.

[19] Ali, A.R., "Micro-optical vibrometer/accelerometer using dielectric microspheres," Appl. Opt. 58, 42114219, 2019.

[20] Ali, A.R., Badawi, H. H., and Algohary, M., "Acoustic sensor based on fiber-optic polymeric resonator," J. Opt. Soc. Am. B 36, 509-516, 2019.

[21] Ali, A.R., Ötügen, V., and Ioppolo, T., "High data rate transient sensing using dielectric micro-resonator", Appl. Opt., 54, 7076-7081, 2015.

[22] Floris, I., Adam, J. M., Calderón, P. A., Sales, S.,"Fiber Optic Shape Sensors: A comprehensive review” Optics and Lasers in Engineering, 139, 106508, 2021. 


\section{NOVEL SENSORS OF THE LINEAR DISPLACEMENT MEASUREMENT WITH HIGH- RESOLUTION FOR MECHATRONICS APPLICATIONS}

[23] Gorodetsky, M. L., Savchenkov, A. A., and Ilchenko, V. S., "Ultimate Q of optical microsphere resonators," Opt. Lett. 21, 453-455, 1996.

[24] Ali, A.R., Afifi, A. N., and Taha, H., "Optical signal processing and tracking of whispering gallery modes in real-time for sensing applications," Proc. SPIE 10249, 102490E, 2017.

[25] Arnold, S., Khoshsima, M., Teraoka, I., Holler, S., and Vollmer, F., "Shift of whispering gallery modes in microspheres by protein adsorption," Opt. Lett. 28, 272-274, 2003.

[26] Ioppolo, T., Ayaz, U. K., and Ötügen, M. V., "Tuning of whispering gallery modes of spherical resonators using an external electric field," Opt. Express 17, 16465-16479, 2009.

[27] Vollmer, F., Brown, D., Libchaber, A., Khoshima, M., Teraoka, I., and Arnold, S., "Protein detection by optical shift of a resonant microcavity," Appl. Phys. Lett. 80, 4057-4059, 2002.

[28] Ioppolo, T., and Ötügen, V., "Pressure tuning of whispering gallery mode resonators," J. Opt. Soc. Am. B 24, 2721-2726, 2007.

[29] Guan, G., Arnold, S., and Ötügen, V., "Temperature measurements using a micro-optical sensor based on whispering gallery modes," AIAA J. 44, 2385-2389, 2006.

[30] Cai, L., Pan, J., Hu, S., "Overview of the coupling methods used in whispering gallery mode resonator systems for sensing" Optics and Lasers in Engineering, 127, 105968, 2020.

[31] Shi, L., Wang, Q., Luo, J., Zhu, T., "In-fiber wavelength-selective reflector based on Y-junction coupled whispering gallery mode resonator" Optics and Lasers in Engineering, 137, 106329, 2021.

[32] Ali, A.R., Ioppolo, T., and Ötügen, M. V., "High-resolution electric field sensor based on whispering gallery modes of a beam-coupled dielectric resonator," International Conference on Engineering and Technology (ICET), Cairo, pp. 1-6, 2012.

[33] Mitu, S. A., Ahmed, K., Al Zahrani, F. A., Grover, A., Rajan, M. S. M., Moni, M. A., "Development and analysis of surface plasmon resonance based refractive index sensor for pregnancy testing" Optics and Lasers in Engineering, 140, 106551, 2021.

[34] Beer, F., Johnston, E., Dewolf, Jr, J., and Mazurek, D., Mechanics of Materials 6 ${ }^{\text {th }}$ ed. McGraw-Hill, 2012.

[35] A.V. Saetchnikov, A.V., Tcherniavskaia, E. A., Saetchnikov, V. A., and Ostendorf, A., " A laser written 4D optical micorcavity for advanced biochemical sensing in aqueous environment"; Journal of Lightwave Technology, Vol. 38, No.8, 2020. 